\title{
Production of Light Nuclei at Thermal Freezeout in Heavy-Ion Collisions
}

\author{
Xinyuan $\mathrm{Xu}^{1,2}$ and Ralf Rapp ${ }^{1}$ \\ ${ }^{1}$ Cyclotron Institute and Department of Physics \& Astronomy, \\ Texas AEM University, College Station, Texas 77843-3366, U.S.A. \\ ${ }^{2}$ Department of Physics, University of Science and Technology of China, Hefei, Anhui 230026, China
}

(Dated: September 13, 2018)

\begin{abstract}
We revisit the problem of the production of light atomic nuclei in ultrarelativistic heavy-ion collisions. While their production systematics is well produced by hadro-chemical freezeout at temperatures near the QCD pseudo-critical temperature, their small binding energies of a few $\mathrm{MeV}$ per nucleon suggest that they cannot survive as bound states under these conditions. Here, we adopt the concept of effective chemical potentials in the hadronic evolution from chemical to thermal freezeout (at typically $T_{\mathrm{fo}} \simeq 100 \mathrm{MeV}$ ), which, despite frequent elastic rescatterings in hadronic matter, conserves the effective numbers of particles which are stable under strong interactions, most notably pions, kaons and nucleons. It turns out that the large chemical potentials that build up for antibaryons result in thermal abundances of light nuclei and antinuclei, formed at thermal freezeout, which essentially agree with the ones evaluated at chemical freezeout. Together with their transverse-momentum spectra, which also indicate a kinetic freezeout near $T_{\text {fo }}$, this provides a natural explanation for their production systematics without postulating their survival at high temperatures.
\end{abstract}

PACS numbers:

\section{INTRODUCTION}

The main goal of ultrarelativistic heavy-ion collisions (URHICs) is, broadly speaking, the production and characterization of hot and dense QCD matter, thereby recreating conditions akin to the first few microseconds in the evolution of our Universe. The current picture of the time evolution of a central URHIC is as follows: a short pre-equilibrium phase (of duration $\Delta \tau_{\text {pre }} \lesssim 0.5 \mathrm{fm} / c$ ) is followed by hydrodynamically expanding quark-gluon plasma $\left(\mathrm{QGP}, \Delta \tau_{\mathrm{QGP}} \simeq 5 \mathrm{fm} / c\right)$, hadronization with a chemical freezeout of the stable hadron species at $T_{\mathrm{H}} \simeq T_{\mathrm{ch}} \simeq 160 \mathrm{MeV}$, followed by a hadronic phase $\left(\Delta \tau_{\text {had }} \simeq 5-10 \mathrm{fm} / c\right)$ with strong elastic collisions (mostly resonance scattering) which maintain approximate local thermal equilibrium until a freezeout temperature of about $T_{\mathrm{fo}} \simeq 100 \mathrm{MeV}$. By that time the fireball has acquired average expansion velocities in excess of half the speed of light, as deduced from systematic analyses of transverse-momentum spectra of pions, kaons and protons [1, 2. In this context, the production of light nuclei and antinuclei has received considerable attention in recent years, see Refs. 3, 4, for a recent reviews. On the one hand, URHICs have enabled the first man-made production of antinuclei beyond nuclear number $A=3$. On the other hand, it was found that the production ratios of the observed nuclei, which by now encompass anti/deuterons, anti- $/{ }^{3} \mathrm{He}$ and anti- $/{ }^{4} \mathrm{He}$ (as well as light hyper anti-/nuclei), closely follow the predictions of the statistical hadronization model at chemical freezeout [5, 6]. At the same time, the $P_{T}$ spectra of the nuclei indicate a kinetic decoupling close to thermal freezeout, suggesting that they participate in the elastic interactions of the hadronic phase. This, however, poses a puzzle, as it is rather surprising that composite objects with binding energies that are about an order of magnitude smaller than the ambient temperature can survive in this environment (cf. also Ref. [7] for a recent discussion of this issue). As an alternative, coalescence models have been employed to form the nuclei in the late stages of the evolution, near thermal freezeout [8, 9. While these models can account for finite-size (or quantum) effects in the formation process, they also require extra parameters, e.g., the so-called coalescence parameter $B_{A}$ (fixing the overall normalization), and often involve an assumption of nearcollinearity of the coalescing nucleon momenta (which, strictly speaking, violates energy conservation).

In the present paper we investigate another option which does not involve additional parameters relative to the chemical freezeout point as specified by a temperature $\left(T_{\mathrm{H}}\right.$ and pertinent baryon chemical potential $\left(\mu_{B}^{\mathrm{H}}\right)$. We will adopt the concept of partial chemical equilibrium throughout the hadronic evolution from $T_{\mathrm{H}}$ to $T_{\text {fo }}$ by introducing chemical potentials for the hadrons which are stable under strong interactions [10 14]. This essentially ensures that the observed ratios of strongly stable hadrons (including strong feeddown contributions) are conserved in the elastic interactions which maintain local thermal equilibrium throughout the hadronic phase. An important variation of this theme, critical for the present analysis, is the separate conservation of baryon $(B)$ and antibaryon $(\bar{B})$ number [12]. The large (stronginteraction) annihilation cross sections of baryons and antibaryons into multi-meson states, e.g., $N \bar{N} \rightarrow 5 \pi$, naively suggest a decline in the total number of baryons plus antibaryons. However, in the presence of large meson-chemical potentials the backward reaction, e.g., $5 \pi \rightarrow N \bar{N}$, as dictated by detailed balance, can largely uphold the $\bar{B} / B$ ratios created at chemical freezeout [15]. With the such obtained baryon and antibaryon chem- 
ical potentials [12] we predict the production ratios of the light nuclei, and compute their transverse-momentum $\left(P_{T}\right)$ spectra, at thermal freezeout, which mitigates the issue of their survival at high temperatures.

The remainder of this article is organized as follows. In Sec. III we recall the basic elements of partial chemical equilibrium and the construction of the effective chemical potential for mesons and anti-/baryons in the hadronic phase of URHICs. In Sec. III we present our main results for the production ratios of light anti-/nuclei evaluated using the predicted chemical potentials at kinetic freezeout. In Sec. IV we evaluate their $P_{T}$ spectra at the same kinetic freezeout. In Sec. $\mathrm{V}$ we conclude.

\section{CHEMICAL FREEZEOUT AND POTENTIALS}

The concept of hadro-chemical freezeout in the fireball evolution of a heavy-ion collision implies that from this point on the observed hadron production ratios (or yields, upon specifying a single 3 -volume, $V_{\mathrm{FB}}\left(T_{\mathrm{ch}}\right)$, to match the total yields to experiment at a given centrality) do no longer change. This concept has been very successful in the description of the hadron production yields as observed in experiment [16 19]. Typical chemical-freezeout temperatures at ultrarelativistic collision energies are close to the pseudo-critical temperature extracted from lattice QCD (1QCD) at vanishing baryon chemical potential, $T_{\mathrm{ch}} \simeq 160 \mathrm{MeV} \simeq T_{\mathrm{pc}}$, while the pertinent baryon chemical potential, $\mu_{B}^{\text {ch }}$, varies from $\sim 240 \mathrm{MeV}$ at top SPS energy $(\sqrt{s}=17.3 \mathrm{GeV})$ via $\sim 25 \mathrm{MeV}$ at top RHIC energy $(\sqrt{s}=200 \mathrm{GeV})$ to $\lesssim 1 \mathrm{MeV}$ at LHC energies $(\sqrt{s}$ of several $\mathrm{TeV}$ ). The microscopic origin of chemical freezeout is associated with the smallness of the cross section of chemistry-changing inelastic interactions of typically a few mb (e.g., for $\pi \pi \rightarrow K \bar{K})$. On the other hand, quasi-elastic resonant interactions, such as $\pi \pi \rightarrow \rho \rightarrow \pi \pi$ or $\pi N \rightarrow \Delta \rightarrow \pi N$ are up to 2 orders of magnitude larger and thus maintain local thermal equilibrium in the expanding fireball down to kinetic freezeout temperatures of $T_{\mathrm{fo}} \simeq 100 \mathrm{MeV}$. These reactions "conserve" the chemistry (total abundances) of the stable particle (such as pions, kaons, etas, nucleons, etc.) which can be accounted for by effective chemical potentials $\left(\mu_{\pi, K, \eta, N, \ldots}\right)$. Within this scheme, the strong resonances, such as $\rho$ and $\Delta$, which are essential for the thermodynamics of the hadronic medium, acquire effective chemical potentials according to the law of mass action, e.g., $\mu_{\Delta}=\mu_{\pi}+\mu_{N}$ and $\mu_{\rho}=2 \mu_{\pi}$. With decreasing temperature, substantial positive meson chemical potentials build up, compensating the suppression of their numbers relative to the conserved baryon number. They typically reach near 100 (150) $\mathrm{MeV}$ for pions (kaons) at kinetic freezeout under both RHIC and LHC conditions, cf. Fig. 1 (the RHIC results shown here differ from the ones originally shown in Ref. [12] by updating the chemical freezeout temperature from $T_{\mathrm{ch}}=180 \mathrm{MeV}$ to $160 \mathrm{MeV}$ ).



FIG. 1: Temperature dependence of chemical potentials for nucleons (solid lines), antinucleons (dashed lines) and pions (dash-dotted lines) at RHIC (red curves) and the LHC (blue curves). The curves for the nucleon and antinucleon chemical potentials at the LHC (solid and dashed blue, respectively), as well as for the pion chemical potentials at RHIC and the LHC (dash-dotted red and blue, respectively), almost coincide.

An exception to the small inelastic hadronic cross sections is the annihilation of baryons and antibaryons into multi-meson states, with cross sections of up to $100 \mathrm{mb}$ in the low-energy range relevant for a thermal heat bath at temperatures of $T \simeq 150 \mathrm{MeV}$. This suggests substantial annihilation rates of antibaryons in the hadronic phase of the fireball and has raised the question of how to reconcile the observed yields of, e.g., antiprotons with the chemical-freezeout picture. In Ref. [15] it was shown that, in the presence of large meson chemical potentials, the inverse reaction of multi-meson fusion into $B \bar{B}$ largely compensates the $B \bar{B}$ annihilation reactions and thus stabilizes the antibaryon abundances throughout the hadronic evolution of the fireball. In essence, this amounts to yet another effective chemical potential, $\mu_{\bar{B}}^{\text {eff }}$, that separately conserves the antibaryon number so that, e.g., $\mu_{\bar{N}}=-\mu_{N}+\mu_{\bar{B}}^{\mathrm{eff}}$, or $\mu_{\bar{\Delta}}=-\mu_{\Delta}+\mu_{\bar{B}}^{\text {eff }}$. The temperature dependence of the nucleon and antinucleon chemical potentials is also shown in Fig. 1 for both RHIC and LHC conditions. It illustrates the role of the effective antibaryon chemical potential, which reaches close to $800 \mathrm{MeV}$ at the lowest temperatures and results in a near constant antiproton-to-proton ratio, $\bar{p} / p \simeq \exp \left[-\left(\mu_{N}-\mu_{\bar{N}}\right) / T\right] \simeq \exp \left[-\left(2 \mu_{N}+\mu_{\bar{B}}^{\mathrm{eff}}\right) / T\right]$. At RHIC, with $\mu_{N}^{\mathrm{ch}} \simeq 25 \mathrm{MeV}$ at chemical freezeout, the nucleon and antinucleon chemical potentials are notably different while at the LHC, where $\mu_{N}^{\text {ch }} \simeq 1 \mathrm{MeV}$, they are close to each other. All of them reach around $400 \mathrm{MeV}$ near thermal freezeout. The baryon and antibaryon numbers are separately conserved, and thus their sum as well. This has important phenomenological implications for, e.g., low-mass dilepton spectra where the medium ef- 


\begin{tabular}{|c|c|c|}
\hline (Anti-) Nucleus & $m_{X}[\mathrm{MeV}]$ & $d_{X}$ \\
\hline \hline$d(\bar{d})$ & 1875.6 & 3 \\
\hline$t(\bar{t})$ & 2808.9 & 2 \\
\hline${ }^{3} \mathrm{He}\left({ }^{3} \overline{\mathrm{He}}\right)$ & 2809.3 & 2 \\
\hline${ }^{4} \mathrm{He}\left({ }^{4} \overline{\mathrm{He}}\right)$ & 3728.3 & 1 \\
\hline
\end{tabular}

TABLE I: The masses (second column) and degeneracies (third column) of the light anti-/nuclei (first column) considered in this work.

fects on the the $\rho$ (and $\omega$ ) meson in the hadronic phase are equally driven by interactions with baryons and antibaryons, and are thus sensitive to the total sum of their densities (see, e.g., Ref. [20] for a recent review).

\section{PRODUCTION RATIOS OF ANTI-/NUCLEI}

We are now in position to apply the hadro-chemistry described in the previous section to production ratios of light anti-/nuclei as recently measured in URHICs. In Tab. III we summarize the masses $\left(m_{X}\right)$ and spin degeneracies $\left(d_{X}\right)$ of the pertinent particles. In the following, we simply compute the densities of these particles as a function of temperature and chemical potential,

$$
n_{X}\left(\mu_{X}, T\right)=d_{X} \int \frac{d^{3} P}{(2 \pi)^{3}} f^{X}\left(E_{X} ; \mu_{X}, T\right),
$$

where $E_{X}=\sqrt{m_{X}^{2}+P^{2}}$ is their energy, $\mu_{X}=\mathrm{A} \mu_{N}$ for nucleus $X, \mu_{\bar{X}}=\mathrm{A}\left(-\mu_{N}+\mu_{B}^{\text {eff }}\right)$ for its antinucleus (A: nuclear mass number $)$, and $f^{X}=\exp \left(-\left(E_{X}-\mu_{X}\right) / T\right)$ is the Boltzmann distribution (quantum-statistical corrections can be safely neglected). Neglecting feeddown contributions, we then take suitable ratios of these densities or evaluate total yields by specifying a single 3 -volume at given centrality.

We start our discussion at RHIC energies. The upper panel of Figure 2 shows the antiparticle-to-particle ratios, computed at both chemical and kinetic freezeout, in comparsion to STAR data for anti-/deuterons, anti$/{ }^{3} \mathrm{He}$ and anti- $/{ }^{4} \mathrm{He}$ [21, 22]. The experimental ratios are obtained from invariant yields in $P_{T}$ bins of width $0.25 \mathrm{GeV}$ around $P_{T} / A=0.875 \mathrm{GeV}$. As discussed in Ref. 22, from estimates using blastwave fits, these ratios differ from the $P_{T}$-integrated yield ratios by $\sim 1 \%$ or so. As expected from the construction of the chemistryconserving evolution of the hadronic medium from chemical down to thermal freezeout, the calculated nucleusto-antinucleus ratios vary little throughout the hadronic phase and show fair agreement with the data. For a direct comparison of our calculations to the absolute values of the $P_{T}$-invariant yields, we utilize our blastwave fits discussed in the following section, since larger variations are expected for particles with different masses when going from finite $P_{T}$ bins to $P_{T}$ integrated yields. In this
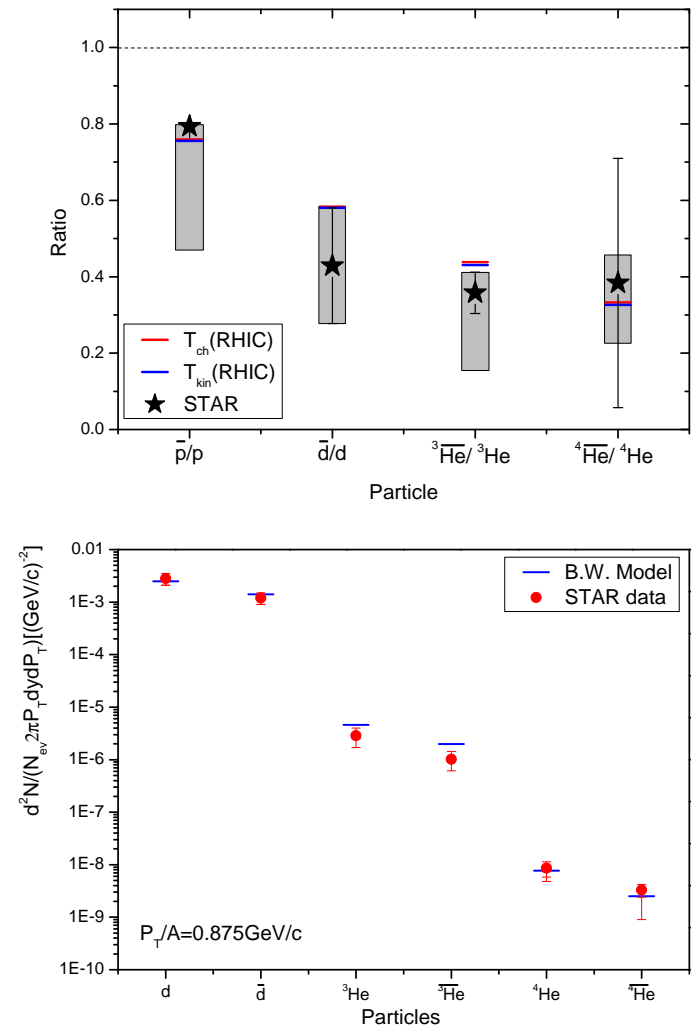

FIG. 2: Production ratios (upper panel) and $P_{T}$-differential invariant yields for $P_{T} / \mathrm{A}=0.875 \mathrm{GeV}$ (lower panel) of light anti-/nuclei in central $\mathrm{Au}+\mathrm{Au}$ collisions at $\sqrt{s_{\mathrm{NN}}}=0.2 \mathrm{TeV}$ at RHIC at chemical (red bars) and kinetic (blue bars) freezeout, compared to STAR data 21, 22.

case, we adjust one overall normalization constant in the anti-/deuteron sector and then predict the yields for anti$/{ }^{3} \mathrm{He}$ and anti- $/{ }^{4} \mathrm{He}$. While the data are reproduced for the latter, a factor of $\sim 2$ discrepancy is observed for the former, see lower panel of Fig. 2, A very similar trend is observed when comparing our model predictions for the $P_{T}$-integrated yields to the recent experimental results of anti-/deuterons and the triton [23, 24]. The origin of this discrepancy remains unclear at this point.

The results for antiparticle-to-particle ratios at the LHC are dislayed in Fig. 3 (upper panel). Again, the calculated results differ little between chemical and kinetic freezeout, and agree with ALICE data within errors. The data for the ratios are compatible with one, reflecting the near baryon-antibaryon symmetric (or netbaryon free) mid-rapidity region at LHC energies. However, turning to the absolute yields (lower panel of Fig. 3), variations over about 6 orders of magnitude between anti/deuterons and anti- $/{ }^{4} \mathrm{He}$ are found, due to the exponential sensitivity to the particle mass. In our calculations, the absolute yields require to fix one volume parameter for a given centrality, which we adjust to the central value of the deuteron yield (we do not include excluded volume corrections here). The remaining five yields for 

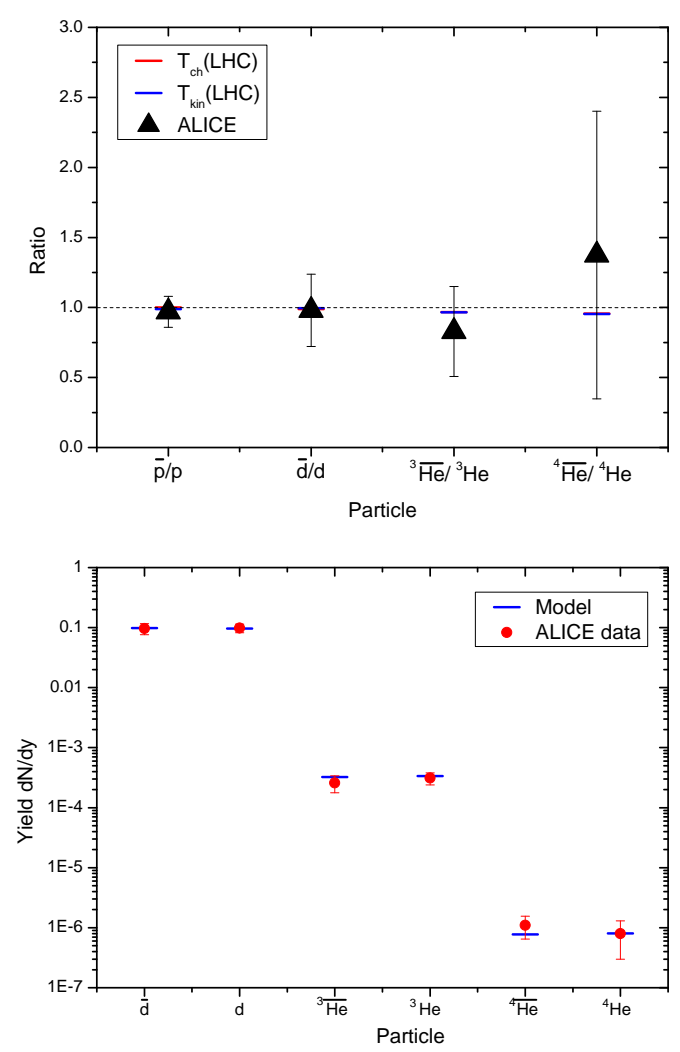

FIG. 3: Production ratios (upper panel) and absolute yields (lower panel) of light anti-/nuclei in $0-10 \%$ central $\mathrm{Pb}+\mathrm{Pb}$ collisions at $\sqrt{s_{\mathrm{NN}}}=2.76 \mathrm{TeV}$ at the LHC at chemical (red bars) and kinetic (blue bars) freezeout, compared to ALICE data 25, 26.

antideuterons, anti- $/{ }^{3} \mathrm{He}$ and anti- $/{ }^{4} \mathrm{He}$ are predictions, which show good agreement with the data. This, of course, has been noted before in statistical-model fits at chemical freezeout [5, 6]; the new point here is that this description is upheld until kinetic freezeout, owing to the conserving chemistry in the hadronic evolution.

\section{TRANSVERSE-MOMENTUM SPECTRA}

To further check the consistency of the description of the production mechanism of light anti-/nuclei at kinetic freezeout, we also revisit their transverse-momentum spectra. Blastwave descriptions have been previously employed for nuclear $P_{T}$ spectra and indeed indicated temperatures and flow velocities near the thermal freezeout extracted from light-hadron $(\pi, K, p)$ spectra. Here, we slightly modify this strategy by fixing the temperature to $T=100 \mathrm{MeV}$ at both RHIC and the LHC and adjust the flow velocity and profile in a common fit to the different anti-/nuclei spectra. We employ the standard blastwave
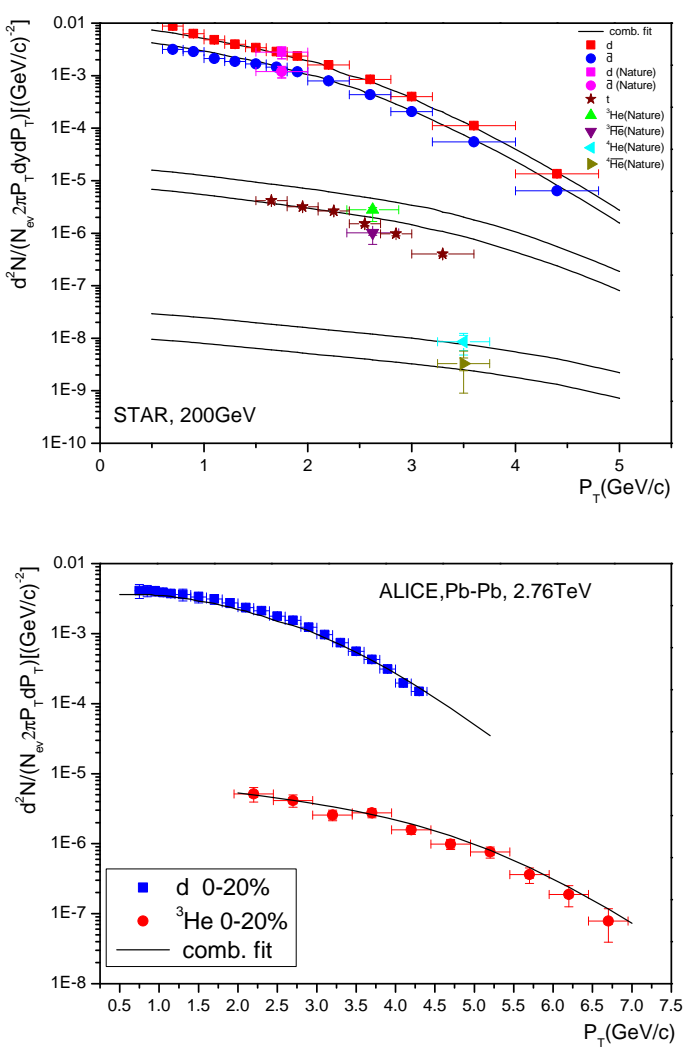

FIG. 4: Transverse-momentum spectra of light anti-/nuclei at RHIC (upper panel) and the LHC (lower panel) in a common blastwave description at kinetic freezeout, compared to STAR data 21-24] for $0-10 \%$ central $\mathrm{Au}+\mathrm{Au}(0.2 \mathrm{TeV})$ collisions and ALICE data 25] for $0-20 \%$ central $\mathrm{Pb}+\mathrm{Pb}(2.76 \mathrm{TeV})$ collsions.

expression [27,

$\frac{d N_{X}}{P_{T} d P_{T}}=C \int_{0}^{R} r d r m_{T} I_{0}\left(\frac{P_{T} \sinh \rho}{T}\right) K_{1}\left(\frac{m_{T} \cosh \rho}{T}\right)$,

with $m_{T}=\sqrt{m_{X}^{2}+P_{T}^{2}}$, a transverse flow rapidity $\rho=$ $\tanh ^{-} 1\left(\beta_{r}\right)$ and flow profile $\beta_{r}=\beta_{s}\left(\frac{r}{R}\right)^{n}$ characterized by the exponent $n$. We find that with a surface flow velocity of $\beta_{s}=0.780 \mathrm{c}$ and $n=1.2$ for central $\mathrm{Au}-\mathrm{Au}$ at RHIC, as well as $\beta_{s}=0.866 c$ and $n=0.8$ for central $\mathrm{Pb}-\mathrm{Pb}$ at the LHC, a fair description of the available $P_{T}$ spectra can be obtained, see Fig. 4. The exception are again the $A=3$ nuclei at RHIC (as found for the yields in the previous section). The larger flow at the LHC is expected due to the longer lifetime the fireball (which, at the same $T$, is larger than at RHIC), and is also in line with the trends extracted from fits to light-hadron spectra [1, 2]. 


\section{CONCLUSIONS}

We have revisited the problem of the production of light nuclei and antinuclei in high-energy heavy-ion collisions. This was triggered by the surprising finding that their production yields follow chemical-freezeout systematics while their small binding energies of a few $\mathrm{MeV}$ per nucleon suggest that these states should not exist at pertinent temperatures of $150-160 \mathrm{MeV}$. We have found that, employing the concept of chemical freezeout in the subsequent hadronic evolution to kinetic freezeout, as previously implemented in different contexts, preserves the ratios and yields of anti-/nuclei evaluated at chemical freezeout down to thermal freezeout (with the exception of $\mathrm{A}=3$ nuclei at RHIC, at both freezeouts). We also found the momentum spectra of the anti-/nuclei at kinetic freezeout to be compatible with a common decoupling with flow velocities extracted from light-hadron spectra. While this enables the formation of the nuclei to occur at much lower temperatures of $T_{\mathrm{fo}} \simeq 100 \mathrm{MeV}$, one can still argue that this is too high for them to exist. However, both the momentum distributions and yields of hadrons are "frozen" at this point and thus should allow for the formation of nuclear bound states even in the aftermath of the hadronic kinetic freezeout, which still conserves the relative chemistry, much like in the evolution from chemical to kinetic freezeout, but without further "cooling" and associated changes in momentum distributions. In other words, the chemical reactions for the for- mation of anti/-nuclei (which are expected to have large cross sections) could continue to still lower densities, to a "nuclear freezeout", without significantly affecting the momentum distributions nor the hadronic chemistry of the hadronic bulk system. This picture appears to be closely related to coalescence mechanisms, but the evaluations are carried out without additional coalescence parameters. After all, coalescence models should, in principle, encode the correct equilibrium limit, an issue that is also pertinent to the hadron formation processes from an equilibrated QGP as well as in the kinetic regime of intermediate transverse momenta [28. We therefore believe that our results can contribute to a better understanding of the formation of light nuclei in URHICs.

\section{Acknowledgment}

We thank Zhenyu Ye and Anton Andronic for help with the STAR and ALICE data, respectively. Some of the ideas of this work have been triggered by discussions at the Terzolas meeting on "Heavy-Ion Physics in the 2020's" (Terzolas, Italy, May 19-21, 2018). This work has been supported by the U.S. National Science Foundation under grant no. PHY-1614484.

\section{Note added}

Shortly before submission of the present ms., a related work appeared 29] discussing the problem of anti/deuteron production at the LHC using a hadronic transport approach.
[1] B. I. Abelev et al. [STAR Collaboration], Phys. Rev. C 79 (2009) 034909

[2] B. Abelev et al. [ALICE Collaboration], Phys. Rev. C 88 (2013) 044910

[3] F. Bellini and A. P. Kalweit, arXiv:1807.05894 [hep-ph].

[4] J. Chen, D. Keane, Y. Ma, A. Tang and Z. Xu, arXiv:1808.09619 [nucl-ex].

[5] A. Andronic, P. Braun-Munzinger, J. Stachel and H. Stocker, Phys. Lett. B 697 (2011) 203

[6] A. Andronic, P. Braun-Munzinger, K. Redlich and J. Stachel, J. Phys. Conf. Ser. 779 (2017) 012012

[7] A. Andronic, P. Braun-Munzinger, K. Redlich and J. Stachel, arXiv:1710.09425 [nucl-th].

[8] J. I. Kapusta, Phys. Rev. C 21 (1980) 1301.

[9] R. Scheibl and U. W. Heinz, Phys. Rev. C 59 (1999) 1585

[10] H. Bebie, P. Gerber, J.L. Goity and H. Leutwyler, Nucl. Phys. B378 (1992) 95.

[11] C.M. Hung and E. Shuryak, Phys. Rev. C57 (1998) 1891.

[12] R. Rapp, Phys. Rev. C 66 (2002) 017901.

[13] D. Teaney, nucl-th/0204023.

[14] T. Hirano and K. Tsuda, Phys. Rev. C 66 (2002) 054905

[15] R. Rapp and E. V. Shuryak, Phys. Rev. Lett. 86 (2001) 2980

[16] P. Braun-Munzinger, K. Redlich and J. Stachel, In *Hwa, R.C. (ed.) et al.: Quark gluon plasma* 491-599

[17] F. Becattini, J. Manninen and M. Gazdzicki, Phys. Rev.
C 73 (2006) 044905

[18] J. Cleymans, H. Oeschler, K. Redlich and S. Wheaton, Phys. Rev. C 73 (2006) 034905

[19] A. Andronic, P. Braun-Munzinger and J. Stachel, Phys. Lett. B 673 (2009) 142 [Erratum: Phys. Lett. B 678 (2009) 516]

[20] R. Rapp, Adv. High Energy Phys. 2013 (2013) 148253

[21] B. I. Abelev et al. [STAR Collaboration], arXiv:0909.0566 [nucl-ex].

[22] H. Agakishiev et al. [STAR Collaboration], Nature 473 (2011) 353 Erratum: [Nature 475 (2011) 412]

[23] N. Yu [STAR Collaboration], Nucl. Phys. A 967 (2017) 788

[24] P. Liu [STAR Collaboration], proceedings of Quark Matter 108 conference (Venice, Italy, May 14-19).

[25] J. Adam et al. [ALICE Collaboration], Phys. Rev. C 93 (2016) no.2, 024917

[26] S. Acharya et al. [ALICE Collaboration], Nucl. Phys. A 971 (2018) 1

[27] E. Schnedermann, J. Sollfrank and U. W. Heinz, Phys. Rev. C 48 (1993) 2462

[28] L. Ravagli and R. Rapp, Phys. Lett. B 655 (2007) 126

[29] D. Oliinychenko, L. G. Pang, H. Elfner and V. Koch, arXiv:1809.03071 [hep-ph]. 